\title{
Point Singularities of Coupled Gauge Fields with Low Energy
}

\author{
T. H. Otway^ and L. M. Sibner ${ }^{\star \star}$ \\ Department of Mathematics, University of Pennsylvania, Philadelphia, PA 19104, USA
}

\begin{abstract}
Isolated singularities in higher-dimensional Yang-Mills-Higgs fields are considered. The singularities are removable if the energy is smaller than a dimensional constant.
\end{abstract}

\section{Introduction}

Conditions under which Yang-Mills-Higgs fields cannot have isolated singularities are now know for arbitrary dimension [5-11]. In all cases the Yang-Mills field $F$ is required to be an element of the space $L^{n / 2}$, where $n$ is dimension; the Higgs field $\phi$ must satisfy certain $L^{p}$ conditions depending on $n$ and on a physical parameter $\lambda$. (In dimension 2 an additional holonomy condition is necessary [9].) The arguments depend crucially on the conformal invariance of the norm $\|h\|_{n / 2}$, where

$$
h^{2}=|F|^{2}+|\nabla \phi|^{2}+|\phi|^{4}
$$

is the density of the energy integral

$$
E=\int h^{2} .
$$

An example is known of a pure Yang-Mills field $(\phi=0)$ in which $h \in L^{p}$ for any $p<n / 2$ and $h$ is singular. In this example, which is defined by pulling back a bundlevalued $F$ on $S^{n-1}, h(x) \sim$ const $/|x|^{2}$. Thus the energy density tends to infinity near $x=0$ and its $L^{2}$ norm, though finite, is not necessarily small. (The example is due to Uhlenbeck and is reported in [7].)

It is reasonable to ask whether the physically natural condition of low energy is sufficient for removing point singularities. One would then hope to show directly that the asymptotically high energy of Uhlenbeck's example accounts for its nonremovable singularity. It would be sufficient to show that $h$ is in the space $L^{n / 2}$

\footnotetext{
* This work represents part of the author's doctoral dissertation at Polytechnic University of New York

$\star \star$ Research partially supported by NSF Grant DMS-8501419
} 
whenever its $L^{2}$ norm is smaller than a dimensional constant. Then known arguments [7] would apply. Thus we prove

Theorem 1. Suppose $h(y)$ is $C^{\infty}$ in a punctured ball and let the components $(F, \phi)$ of $h$ satisfy there the Yang-Mills-Higgs equations (1.1), (1.2) below. Then there is a constant $\gamma$ depending on dimension $n>4$ and on $\lambda$ such that if $\|h\|_{2 ; B_{1}}<\gamma$ the apparent point singularity is removable.

We note that $h \in C^{\infty}$ obviously implies that both $F$ and $\phi$ are $C^{\infty}$. Here and below \|\|$_{p}$ denotes $L^{p}$ norm.

For dimensions lower than 4 the conditions of the theorem are too strong $[6,8]$. In the case $n=4$, the energy integral is itself conformally invariant, and one can find a conformally Euclidean metric in which any finite-energy field is small. Thus in dimension 4 the theorem follows from previous work $[5,8,10]$.

Liao has proven an analogous result for harmonic maps [3].

In Sect. 1 we briefly review some of the analytic aspects of gauge theory (see [2] for details). We prove the theorem in Sect. 2.

\section{The Euclidean Higgs Model}

Let $\Omega$ be a simply connected domain of $\mathbf{R}^{n}$ and $P$ a principal bundle over $\Omega$ with compact structure group $G$. Form the associated vector bundle $\eta$ and identify $G$ with the bundle automorphism of $\eta$. The Lie algebra $g$ appears as the fiber of the adjoint bundle, ad $\eta=P \times_{G}$ g. There is a local representation of the Yang-Mills connection $A$ on $\eta$ as a 1 -form taking values in $g$. The Yang-Mills curvature $F$ appears as the $\mathrm{g}$-valued 2 -form

$$
F=d A+\frac{1}{2}[A, A],
$$

where $[$,$] denotes Lie bracket.$

Physically $A$ represents the vector potential of a force field $F$. In the Higgs model $F$ interacts with the scalar field $\phi$ induced by a particle of mass $m$. Mathematically the scalar field is a section of $\eta \otimes L$, where $L$ is a line bundle over $\Omega$. Thus under scale changes $y=r x$, we have $\phi(y)=r^{-1} \phi(x)$. Similarly, $m$ is a section of $L$, constant in a fixed coordinate frame and having unit conformal weight under scale changes.

These fields are assumed to satisfy the coupled Yang-Mills-Higgs equations

$$
\left.\begin{array}{l}
D^{*} F=[D \phi, \phi] \\
D^{*} D \phi=\frac{\lambda}{2}\left(|\phi|^{2}-m^{2}\right) \phi,
\end{array}\right\}
$$

where $D=d+[A, \cdot]$, and $\lambda$ is the physical parameter mentioned earlier. [We define $D$ so that the Laplacian of (1.1) and all subsequent Laplacians in this paper have positive principal part.] In addition to the two field equations (1.1) there is a third equation, the Bianchi identity

$$
D F=0,
$$

which is satisfied by any curvature form. 
Equations (1.1) are the Euler-Lagrange equations of the action integral

$$
a=\frac{1}{2} \int_{\mathbf{R}^{n}}\left\{|F|^{2}+|\nabla \phi|^{2}+\frac{\lambda}{4}\left(|\phi|^{2}-m^{2}\right)^{2}\right\} .
$$

The problem is to remove a possible point singularity (say, at the origin) from a $C^{\infty}$ solution of (1.1), (1.2) on a bundle $\eta$ over a punctured ball centered at the origin. We say that the singularity in the coupled Yang-Mills field is removable if the configuration $(A, F, \phi)$ is gauge equivalent to a $C^{\infty}$ configuration on a (topologically identical) bundle over the entire ball, including the origin. Two configurations $\left(A_{1}, F_{1}, \phi_{1}\right)$ and $\left(A_{2}, F_{2}, \phi_{2}\right)$ are said to be gauge equivalent if they are related by a continuous map $s: \Omega \rightarrow G$ such that

$$
A_{2}=s^{-1} A_{1} s+s^{-1} d s, \quad F_{2}=s^{-1} F_{1} s, \quad \phi_{2}=s^{-1} \phi_{1} s .
$$

The idea of the proof is to show that the elements of some arbitrary original configuration are in a sufficiently high $L^{p}$ space so that the gauge transformation to a smooth configuration will be continuous (topology-preserving). This idea originally appeared in $[10,11]$.

\section{The Proof}

Since $h$ satisfies, for nonnegative constants $a$ and $b,[8]$

$$
\Delta h+(a h+b) h \geqq 0
$$

(where the Laplacian $\Delta=\sum_{i} \partial_{i} \partial_{i}$ ), we can apply Morrey's lemma [4, Theorem 5.3.1] in the reference annulus $V=\left\{y\left|\frac{1}{2} \leqq\right| y \mid \leqq 1\right\}$ to obtain, for $|y|=3 / 4$,

$$
h(y) \leqq \tilde{c}\|h\|_{2 ; V} \leqq \tilde{c}\|h\|_{2 ; B_{1}}
$$

where $\tilde{c}$ is the constant in Morrey's lemma. It follows by scaling that for $x=\frac{4}{3} y r$,

$$
|x|^{2} h(x) \leqq c \gamma .
$$

This implies that $h \in L^{p}$ for any $p<n / 2$.

To show that an apparent point singularity at $x=0$ is removable it is sufficient [7] to show that

$$
\int_{\varepsilon<|x|<R}|h(x)|^{n / 2} d x \leqq K<+\infty
$$

for $K$ independent of $\varepsilon$. We proceed as in Gidas and Spruck [1], noticing first that by (3.3)

$$
\int h^{n / 2} d x=\int h \cdot h^{(n-2) / 2} d x \leqq c^{\prime}(\gamma) \int h \cdot r^{2-n} d x .
$$

We have, for nonnegative $\xi \in C_{0}^{\infty}(\Gamma), \Gamma=\{x|\varepsilon<| x \mid<R\}, R$ small,

$$
\int_{\Gamma} \nabla \xi \cdot \nabla h \leqq \int_{\Gamma} \xi(a h+b) h .
$$


Choose $\xi(x)=\zeta(x) \psi(x)$, where

$$
\begin{aligned}
& \zeta(x)=\left\{\begin{array}{lll}
1 & \text { for } & 2 \varepsilon \leqq|x| \leqq R / 2, \\
0 & \text { for } & |x| \leqq \varepsilon,|x| \geqq R,
\end{array}\right. \\
& \|\nabla \zeta\|_{\infty} \leqq k / \varepsilon, \quad\|\Delta \zeta\|_{\infty} \leqq k^{\prime} / \varepsilon^{2}
\end{aligned}
$$

and

$$
\psi(x)=|x|^{4-n}
$$

Then (3.6) and (3.3) imply

$$
\begin{aligned}
& \int_{\Gamma} \zeta h \psi\left[-\frac{\Delta \psi}{\psi}-(a h+b)\right] \leqq \int_{\Gamma}[\psi(\Delta \zeta)+2 \nabla \zeta \cdot \nabla \psi] h \\
& =\int_{\varepsilon<|x|<2 \varepsilon}[\psi(\Delta \zeta)+2 \nabla \zeta \cdot \nabla \psi] h+\int_{\frac{1}{2} R \leqq|x|<R}[\psi(\Delta \zeta)+2 \nabla \zeta \cdot \nabla \psi] h \\
& \quad \leqq K<\infty
\end{aligned}
$$

(since $\zeta$ is constant on $2 \varepsilon \leqq|x| \leqq R / 2$ ), where $K$ is independent of $\varepsilon$. Note that

$$
a h+b \leqq\left(c a \gamma+R^{2} b\right) / r^{2} \text {, }
$$

and

$$
-\Delta \psi / \psi=2(n-4) / r^{2} \text {. }
$$

Hence if $R$ is sufficiently small, $\gamma$ can be chosen so small that $2(n-4)-($ ca $\gamma$ $\left.+R^{2} b\right)>0$. Thus

$$
-\Delta \psi / \psi-(a h+b) \geqq K^{\prime} / r^{2}
$$

with $K^{\prime}$ positive and independent of $\varepsilon$. Substituting (3.9) into the left-hand side of (3.7) and comparing this with (3.5) as $\zeta \rightarrow 1$, we see that $\|h\|_{n / 2}$ is bounded independently of $\varepsilon$. This completes the proof.

Note added in proof. There is another method for obtaining the a priori estimate (3.3). It is essentially due to R. Schoen and K. Uhlenbeck [see Schoen's article in: Seminar in nonlinear partial differential equations, S. S. Chern (ed.), Springer 1984]. The method has been extended to pure Yang-Mills fields by H. Nakajima and, independently, by F. Duzaar (preprint). The crucial fact in generalizing such results to the Yang-Mills Lagrangian is the covariance of this functional under diffeomorphisms of the underlying domain which lift to automorphisms of the principal bundle [Parker, T.: Math. Z. 185, 305-314 (1984); Price, P.: Manuscripta Math. 43, 131-166 (1983)]. Replacing the energy $E$ by the action $a$ throughout, one obtains a monotonicity formula and (3.3) follows.

Acknowledgements. The authors would like to thank B. Gidas and R. Schoen for helpful discussions.

\section{References}

1. Gidas, B., Spruck, J.: Global and local behavior of positive solutions of nonlinear elliptic equations. Commun. Pure Appl. Math. 34, 525-598 (1981)

2. Jaffe, A., Taubes, C.: Vortices and monopoles. Boston: Birkhäuser 1980

3. Liao, G.: A regularity theorem for harmonic maps with small energy. J. Differ. Geom. 22, 233-241 (1985)

4. Morrey, C.B.: Multiple integrals in the calculus of variations. Berlin, Heidelberg, New York: Springer 1966 
5. Parker, T.H.: Gauge theories on four dimensional Riemannian manifolds. Commun. Math. Phys. 85, 563-602 (1982)

6. Sibner, L.M.: Removable singularities of Yang-Mills fields in $\mathbf{R}^{3}$. Compos. Math. $\mathbf{5 3}, 91-104$ (1984)

7. Sibner, L.M.: The isolated point singularity problem for the coupled Yang-Mills equations in higher dimensions. Math. Ann. 271, 125-131 (1985)

8. Sibner, L.M., Sibner, R.J.: Removable singularities of coupled Yang-Mills fields in $\mathbf{R}^{3}$. Commun. Math. Phys. 93, 1-17 (1984)

9. Smith, P.D.: A removable singularities theorem for the coupled Yang-Mills-Higgs equations in two dimensions. Preprint

10. Uhlenbeck, K.K.: Removable singularities in Yang-Mills fields. Commun. Math. Phys. 83, 11-29 (1982)

11. Uhlenbeck, K.K.: Connections with $L^{p}$ bounds on curvature. Commun. Math. Phys. 83, 31-42 (1982)

Communicated by C. H. Taubes

Received October 21, 1986; in revised form February 2, 1987 
